\title{
To Study the Correlation of Nerve Conduction Velocity Studies and The Outcome of Subjects with Bell's Palsy on Sunnybrook Facial Grading Scale
}

\author{
Neha Patel ${ }^{* 1}$, Pragna Landge ${ }^{2}$.
}

${ }^{{ }_{1}}$ Adult Neurological and Psychosomatic Disorders, Neuro Physiotherapist, Gurugram, Haryana, India.

${ }^{2}$ Adult Neurological and Psychosomatic Disorders, Assistant Professor, Krishna school of Physiotherapy and Rehabilitation, Varnama, Vadodara, India.

\section{ABSTRACT}

Background: Due to limited literature, on the usage of Sunnybrook Facial Grading scale (SFGS), we attempt to address SFGS in measuring clinical outcomes and understand its correlation with NVCs to predict the recovery course.

Objectives: 1. To correlate the findings of Nerve Conduction Velocity studies on SFGS in subjects with Bell's palsy. 2. Also based on the correlation to find whether Nerve Conduction Velocity studies can be used as a prognostic indicator/tool in Bell's palsy.

Methodology: A total of 58 subjects with unilateral Bell's palsy were selected for the study. Outcome measures, SFGS and NCVs were evaluated on the first day and $20^{\text {th }}$ day.

Results: Karl Pearson's correlation coefficient showed a strong positive correlation between the amplitude of frontalis and mentalis muscle.

Conclusion: A positive correlation of the amplitude of frontalis and mentalis muscle.

KEYWORDS: Nerve Conduction velocity studies, Sunnybrook Facial Grading scale, Bell's palsy, correlation and prognostic tool.

Address for correspondence: Neha Patel, MPT, Adult Neurological and Psychosomatic Disorders, Neuro Physiotherapist, Gurugram, Haryana, India. E-Mail: smile.neh88@gmail.com

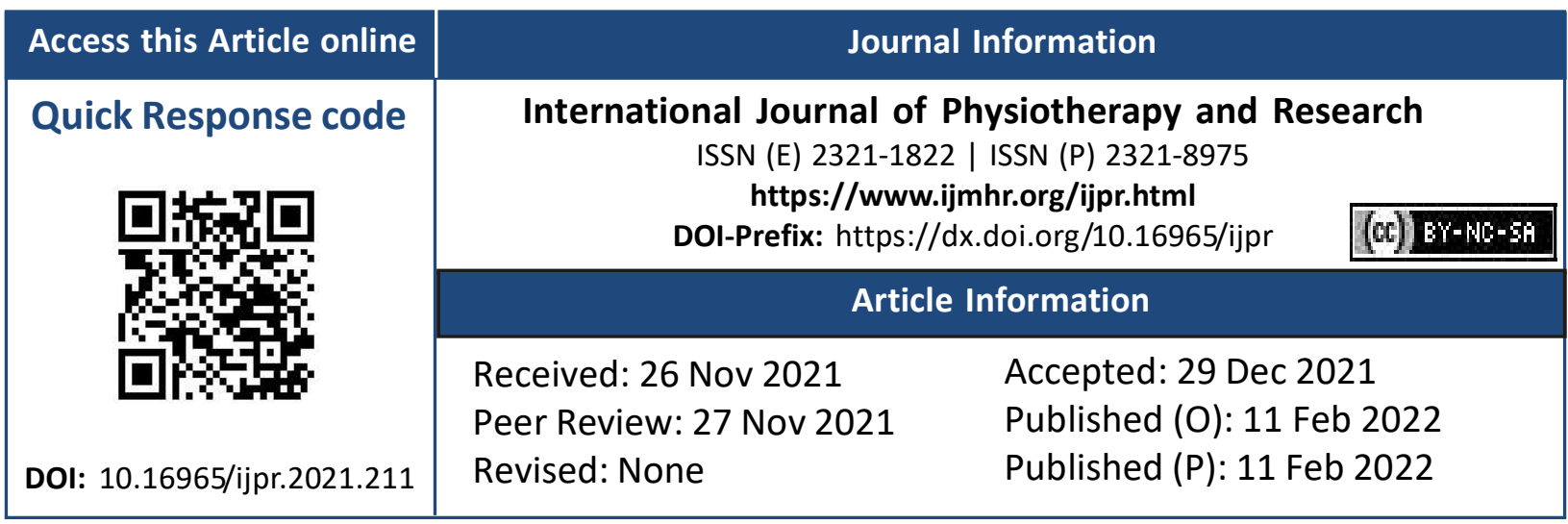

\section{INTRODUCTION}

Neural injuries are functionally disabling and they lack a complete recovery in terms of function and quality of life to normal. The investigative procedures and clinical outcome measures are always taken into consideration for reaching an objective of better prediction of outcome and studies are being carried out to establish a correlation between them. Neural system affection poses a challenge in diagnosis, understanding the causative factors, and developing a therapy strategy.

Bell's palsy is an idiopathic, acute peripheralnerve palsy involving the facial nerve, which supplies all the muscles of facial expression [1]. Bell's palsy is probably the single most common cause of facial nerve disorders [2]. The time from onset of Bell's palsy to total, unilateral facial paralysis is usually about 24 to 48 hours. Spontaneous recovery is common, occurring among some $75 \%$ to $80 \%$ of all patients, usually in about 3 to 4 weeks. 
However, about $15 \%$ to $20 \%$ of patients maintain a lifelong residual weakness following resolution of the Bell's palsy. About $5 \%$ of all patients have permanent weakness [2].

Classically, Bell's palsy has been defined as idiopathic, and the cause of the inflammatory process in the facial nerve remains uncertain[1].

Patients with incomplete palsy have a better prognosis than patients with complete palsy and the younger the patient the better the prognosis $[3,4]$. The prognosis of Bell's palsy is related to the severity of the lesion. A simple rule is that clinically incomplete lesions tend to recover. Therefore, complete injuries may not recover fully.

The Sunnybrook scale, proposed by Ross et al [5], is a weighted subjective scale that incorporates primary and secondary defects in the calculation of a single score. The authors reported that this approach was able to distinguish between levels of facial nerve dysfunction, which was not distinguishable with the House-Brackmann scale [6]. And therefore, this scale was chosen in the present research over the House-Brackmann scale. The Sunnybrook Facial Grading System (SFGS) is a well-established tool for assessing facial movement outcomes [4].

This tool should be clinically relevant and easy to administer, provide a quantitative score for reporting purposes, and be sensitive enough to detect clinically important changes over time or with treatment. Different regions of the face are examined separately with the use of five standard expressions [5-11].

Serial electrodiagnostic studies help delineate the course of the illness. The amplitude of the direct response elicited by stimulation of the facial nerve after the fourth to the fifth day of onset serves as the best means of predicting the eventual outcome of recovery [12].

Nerve Conduction Velocity studies help localize and characterize a focal lesion if conducted as an extension of a physical examination in a proper clinical context. They may provide the evidence of conduction abnormalities [7]. With steady improvement and standardization of methods, they have become a reliable test in clinical settings and are now widely used not only for precise localization of a lesion, but also for accurate characterization of the nerve function [8].

A facial nerve conduction study in Bell's palsy provides a good reflection of the prognosis, and the degree of nerve damage also [9].

Early publications by Fisch address the use of ENoG which includes NCVs and EMG, in Bell's palsy patients. He readily admits the test's utility is not universal. Testing is restricted to the acute phase of Bell's palsy, as Fisch found the correlation between denervated facial muscles and degenerated motor nerve fibers most accurate within the first 2 to 3 weeks after onset. Additionally, ENoG cannot differentiate between degrees of nerve damage and as a comparative modality is only useful in cases of unilateral paralysis. Even with these limitations, Fisch's work showed ENoG has excellent prognostic qualities concerning Bell's palsy outcomes [10]. Electroneuronography is found to be valuable in the prognostic evaluation [11].

The electrodiagnosis comparative study of \%CMAP amplitude between the affected and the sound side has an important role in determining the prognosis. If the amplitude was found higher than 10\%CMAP in the affected side, the patient had a good chance for functional recovery. The percentage of CMAP amplitude could be used for predicting recovery of facial paralysis from Bell's palsy and other causes [12-15].

Despite certain limitations, NCVs can provide diagnostically pertinent information if used judiciously in appropriate clinical contexts [8].

There is a constant need for better objective and reproducible methods for evaluating facial paralysis led to the advent of new technology directed at the evaluation of facial function. The need for the correlation exists and improvement in ability to predict better outcome will help in reducing the stress of uncertainty of recovery.

The prognostic value of electrophysiology and its significance in the prediction facial function outcome in this field remains unclear because of the different methodologies used. 
Prognosis can be determined, and excessive tests can be eliminated with proper timing and electrophysiological testing [16]. These tests may bridge the gap between the onset of the palsy and the period in which other electrodiagnostic tests can be usefully employed.

Advances in various fields of medicine along with the electrodiagnostic testing and rehabilitation of the facial nerve indicates that the most important tenet for all in treatment is proper diagnosis and early treatment [18]. Thus, Nerve conduction studies can obtain with ease, the results, thereby also enhancing the early treatment requirement. Being applied to a large population, still its clinical utility is not known [19]. Hence, the need arises for establishing a correlation between the NCVs and the outcome measure i.e., SFGS in patients in the Bell's palsy and proving it to be of a prognostic value, especially in India.

\section{METHODOLOGY}

\section{MATERIALS USED:}

1. Nerve Conduction Velocity Equipment with a monitor (Biotech Neurocare 2000).

2. Printer

3. Sterilizer

4. Electrode gel

5. Water

6. A micro-pore tape

7. Cotton

8. A pencil

9. Paper

10. Sunnybrook Facial Grading scale (SFGS). Inclusion Criteria:

1. Patient already diagnosed with unilateral Bells Palsy and reporting to physiotherapy department within one week of onset.

2. The patients have sufficient physical and mental ability to understand the instructions and to cooperate throughout the session.

3 . The age of the patient should be between $30-70$ years.

\section{Exclusion criteria:}

1. Patients with a previous history of peripheral facial paralysis, Ramsay Hunt syndrome, traumatic facial paralysis and central or peripheral nervous system disease other than IPFP will be excluded.

2. Patients with Facial Palsy (supra-nuclear lesions or any known cause of the infection).

3. Patients with any history or trauma or fracture of the skull bone.

4. Patients with diabetes mellitus.

5. Patients who have undergone a recent facial surgery.

6. Patients with any skin allergic problems, especially on the face.

7. Any contraindications for NCVs, like presence of metal implants.

Procedure: Ethical Clearance has been obtained from S.D.M. college of Medical Science And Hospital. A total of 58 patients diagnosed with unilateral Bell's palsy diagnosed by a physician/ENT specialist were screened as per inclusion and exclusion criteria were included in the research. Patient willing to participate were briefed about the research and their written consent were taken.

The Sunnybrook Facial Grading Scale (SGFS) is used to measure the muscle function at Rest (3 components), during a voluntary movement (5 components), and the effect of synkinesis (4 components).

The scores of the SFGS range from 0 (complete paralysis) to 100 (normal facial function). The 3 sections to the SFGS-resting posture (SFGS rest), voluntary movement (SFGS movement), and synkinesis (SFGS synkinesis)-are scored individually, and the scores are combined for a total or composite score. For both the SFGS rest and SFGS synkinesis sections, a higher score relates to greater impairments. For the SFGS movement section, a lower score relates to greater impairment. The SFGS score is calculated as follows: SFGS= SFGS movement" SFGS rest" SFGS synkinesis.

All the subjects with Bell's palsy who were seen within 1 week of the onset (acute phase) were included in the study and the SFGS was noted.

The subjects were clearly explained about the procedure of Nerve Conduction Velocity studies. Recording/surface electrodes are placed at Frontalis, and Mentalis; upper and lower 
trunk respectively. And the ground electrode is placed anywhere between the cathode and the stimulating site. The stimulating electrode is placed at the stylomastoid foramen, just beneath the ear.

The waveform of the maximum amplitude with the minimal intensity is selected. The same procedure is repeated for the mentalis muscle. On the whole, the full procedure is first done on the normal side of the face and then the same was repeated on the affected side of the face.

On the second visit, 20th day after the onset (subacute phase) the NCV was performed to determine the extent of the recovery. Again the outcome of Bells palsy is clinically evaluated using the same grading system (SFGS). The third visit is the follow-up after one month, on the 30th day after the onset, following which the outcome of Bells palsy is clinically evaluated using the same grading system (SFGS).

The scores of SFGS are compared from the readings of the first visit to the second visit of amplitude of NCV graph.

Also the patients would be undergoing conventional physiotherapy treatment which includes electrical stimulation, PNF techniques, taping as required and the facial exercises given as home advice. The patients were continued with the physiotherapy treatment even after the study was over. (Intention to treat analysis)

Data analysis: In this study statistical analysis was done using SPSS (Statistical Package for the Social Sciences) version 20.0. Initially, a demographic analysis was done between the age groups and gender; and, side of affection and gender.
The correlation of amplitude of the frontalis muscle was done with the SFGS pre and postscores using Karl Pearson's correlation coefficient method. The same correlation was used for mentalis muscle comparing the pre and post-test scores.

Multiple regression analysis of pretest SFGS scores by frontalis amplitude and mentalis amplitude scores was done. Following this, a multiple regression analysis of posttest SFGS scores by frontalis amplitude and mentalis amplitude scores was also done.

The Logistic regression analysis of posttest SFGS scores (low and high) by frontalis amplitude (low and high) and mentalis amplitude (low and high) scores was performed to find out the relationship between the frontalis amplitude and the mentalis amplitude variation.

We assumed that $10 \%$ of the amplitude of the normal side would be a predictor of the extent of the severity on the affected side. Also, the comparison of the $10 \%$ amplitude of the normal side (greater than or less than 10\%) was done with the muscle to find out the prognosis of the patients with Bell's palsy.

We also studied the correlation between frontalis and mentalis first reading with a gain of pre and post-test scores of SFGS. This is to predict the prognosis of the patient with Bell's palsy based on this correlation.

Data were presented as mean \pm standard deviation (SD) and percentage (P).

$P$ value $<0.05$ was considered statistically significant.

\section{RESULTS}

Table 1: Distribution of samples by age groups and gender:

\begin{tabular}{ccccccc}
\hline Age groups & Male & $\mathbf{\%}$ & Female & \% & Total & $\%$ \\
\hline 20-39yrs & 18 & 58.06 & 13 & 41.94 & 31 & 53.45 \\
\hline 40-59yrs & 8 & 47.06 & 9 & 52.94 & 17 & 29.31 \\
\hline 60+yrs & 4 & 40 & 6 & 60 & 10 & 17.24 \\
\hline Total & 30 & 51.72 & 28 & 48.28 & 58 & 100 \\
\hline Mean age & \multicolumn{2}{c}{39.83} & \multicolumn{2}{c}{45.75} & \multicolumn{2}{c}{42.69} \\
\hline SD age & 14.64 & \multicolumn{2}{c}{14.26} & & \\
\hline
\end{tabular}


Table 2: Distribution of samples by side of affected and gender:

\begin{tabular}{ccccccc}
\hline Side of affected & Male & $\%$ & Female & \% & Total & $\%$ \\
\hline Left side & 18 & 62.07 & 11 & 37.93 & 29 & 50 \\
\hline Right side & 12 & 41.38 & 17 & 58.62 & 29 & 50 \\
\hline Total & 30 & 51.72 & 28 & 48.28 & 58 & 100 \\
\hline
\end{tabular}

Table 3: Correlation between frontalis amplitude scores Table 4: Correlation between mentalis amplitude scores with pre and posttest SFGS scores by Karl Pearson's with pre and posttest SFGS scores by Karl Pearson's correlation coefficient method:

\begin{tabular}{|c|c|c|c|c|c|c|c|}
\hline \multirow[t]{2}{*}{ Variables } & \multicolumn{3}{|c|}{$\begin{array}{l}\text { Correlation between frontalis } \\
\text { amplitude scores with }\end{array}$} & \multirow[t]{2}{*}{ Variables } & \multicolumn{3}{|c|}{$\begin{array}{l}\text { Correlation between mentalis } \\
\text { amplitude scores with }\end{array}$} \\
\hline & r-value & t-value & p-value & & r-value & t-value & p-value \\
\hline Pretest SFGS & 0.1318 & 0.9949 & 0.3241 & Pretest SFGS & 0.0034 & 0.0256 & 0.9797 \\
\hline Post-test SFGS & 0.2738 & 2.1304 & $0.0375^{*}$ & Post-test SFGS & 0.2907 & 2.274 & $0.0268^{*}$ \\
\hline
\end{tabular}

Table 5: Multiple regression analysis of pretest SFGS scores by frontalis amplitude and mentalis amplitude scores:

\begin{tabular}{cccccc} 
& Variables & $\begin{array}{c}\text { Regression } \\
\text { coefficient }\end{array}$ & $\begin{array}{c}\text { SE of reg. } \\
\text { Coefficient }\end{array}$ & t-value & p-level \\
\cline { 2 - 6 }$* \mathrm{p}<0.05$ & 33.176 & 3.9976 & 8.299 & $0.00001^{*}$ \\
\cline { 2 - 5 } & Intercept & 0.0647 & 0.0606 & 1.0679 & 0.2902 \\
\cline { 2 - 5 } & Mrontalis amplitude & -0.027 & 0.0663 & -0.4074 & 0.6853 \\
\hline & Mentalis amplitude & $\mathrm{R}=0.1425, \mathrm{R}^{2}=0.0203, \mathrm{~F}(2,55)=.57050 \mathrm{p}=0.56855$ Std.Error of estimate: 9.1502 \\
\hline
\end{tabular}

Table 6: Multiple regression analysis of posttest SFGS scores by frontalis amplitude and mentalis amplitude scores:

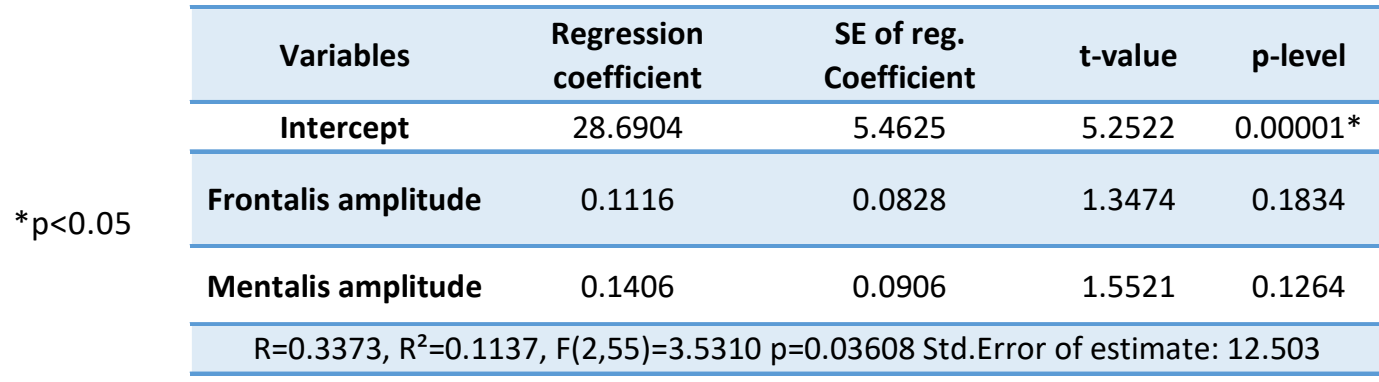

Table 7: Logistic regression analysis of posttest SFGS scores (low and high) by frontalis amplitude (low and high) and mentalis amplitude (low and high) scores:

\begin{tabular}{cccccc}
\hline Variables & Coefficient & Std. Err. & Odd ratio & z-value & p-value \\
\hline Frontalis amplitude & 1.09 & 0.6 & 2.98 & 1.81 & 0.071 \\
\hline Mentalis amplitude & 0.38 & 0.59 & 1.47 & 0.65 & 0.516 \\
\hline
\end{tabular}

Table 8: Comparison of pretest and posttest SFGS scores by paired t test:

\begin{tabular}{|c|c|c|c|c|c|c|c|}
\hline Time & Mean & Std.Dv. & Mean Diff. & SD Diff. & $\%$ of change & Paired $t$ & p-value \\
\hline Pretest & 35.5 & 9.08 & \multirow{2}{*}{-7.02} & \multirow{2}{*}{11.06} & \multirow{2}{*}{-19.77} & \multirow{2}{*}{-4.8318} & \multirow{2}{*}{$0.00001^{*}$} \\
\hline Posttest & 42.52 & 13.05 & & & & & \\
\hline
\end{tabular}

Table 9: Comparison of the 10\% amplitude of the Table 10: Correlation between frontalis and mentalis normal side (greater than or less than $10 \%$ ) with the muscle:

\begin{tabular}{ccc}
\hline \multirow{2}{*}{ Muscle } & \multicolumn{2}{c}{$\mathbf{1 0 \%}$ of amplitude Normal Side } \\
\cline { 2 - 3 } & Greater than $\mathbf{1 0 \%}$ & Less than $\mathbf{1 0 \%}$ \\
\hline Frontalis & 57 & 1 \\
\hline Mentalis & 58 & 0 \\
\hline
\end{tabular}

first reading with gain of pre and post test scores of SFGS:

\begin{tabular}{cccc}
\hline Variables & r-value & t-value & p value \\
\hline Frontalis & -0.0496 & -0.2939 & 0.7706 \\
\hline Mentalis & 0.5595 & 3.994 & $\mathbf{0 . 0 0 0 3 *}^{*}$ \\
\hline
\end{tabular}




\section{DISCUSSION}

The purpose of this study was to correlate the findings of the Nerve Conduction Velocity studies on Sunnybrook Facial Grading Scale (SFGS) in the subjects with Bell's palsy and understand the ability to objectively predict the outcome.

A factor that impinges on recovery rates is the method used to assess facial function; grading facial function from photos or videos has a lower sensitivity for minor sequelae and most probably results in higher recovery rates compared with live grading. ${ }^{20}$ Therefore, we used a more standard facial grading scale i.e., SFGS in our study. The Sunnybrook facial grading shows a composite score from 0 to 100 , where 0 is complete paralysis and 100 indicates normal function. The higher sensitivity of the Sunnybrook system for minor sequelae most probably explains the lower recovery rates with this grading system compared with those obtained with House-Brackmann. The regional weighted Sunnybrook scale succeeds in reporting results in a more continuous manner and with a wider response range than the gross HouseBrackmann grading scale [5].

Nerve conduction studies, by providing an objective quantitative assessment of facial nerve function, are potentially the most accurate method of assessing facial nerve degeneration. The reduction in amplitude of the CMAP on the affected side, when compared with the normal side is thought to reflect the number of fibers that have undergone Wallerian degeneration. The conduction velocity also can be altered at an initial acute stage of Bell's palsy, in this study our main focus was on amplitude keeping in mind the number of functioning myelinated fibers and its overall diameter. The amplitude of the wave form is a more valid measure than considering conduction velocity as it includes the conduction time of the impulse through neuromuscular junction and we cannot take a second reading to calculate the pure motor nerve conduction. Hence, we considered the amplitude of facial nerve waveform.

Facial nerve conduction studies were used by
Fisch, Erik Stalberg, Mats Engström, May, P. Kokotis. Amongst them, Fisch measured the CMAP amplitude ratio of the affected to the unaffected side, a ratio of less than $10 \%$ being an index of poor recovery. We have used a similar index in our study. In addition, another method is by using the $75 \%$ cut-off point suggested by Ozgur A et al. [16], and also an amplitude greater than one half of the control value on the normal side indicates a good prognosis, although late degeneration can still occur. ${ }^{7}$ Hence, we used less than $10 \%$ for the recovery index as we thought a ratio of $10 \%$ would give us better chances of prognosis at an initial stage in bell's palsy.

The subjects were undergoing physical therapy treatment for Bell's palsy simultaneously in our Physiotherapy department. We assume that the treatment may have a beneficial effect and facilitate the recovery process and the overall outcome of the patient. Thus, it also might have influenced the amplitude variation on the NCV readings and the SFGS scores.

To predict the outcome of the patients we correlated frontalis and mentalis amplitude first reading with the gain of pre and post-test SFGS scores.

As shown in table 1 , our study represents the relation of age group and gender in our subjects. In this study, we have taken an age group of 30 to 70 years of age. In our study majority of the patients are between 30 years to 39 years of age, i.e. 18 males $(58 \%)$ and 13 females (41\%). A total of 31 out of 58 subjects were in $20-39$ years of age group (53.45\%). Several studies have found that increasing age was associated with poorer outcome [21-24].

Based on our data; we found that older age groups had a lesser gain in the SFGS scores than the younger age groups. Age can be a weak risk factor for recovery in Bell's palsy but may not be the major risk factor. As for the gender, our study showed no significant difference between males and females in the incidence of Bell's palsy.

Table 2 shows a comparison between the side affected and gender amongst all 58 subjects. Thereby making a total of 30 males (51.72\%) 
and 28 females (48.28\%). This result is in agreement with the data from Bell's palsy by Donald Gilden that left and right sides of the face are involved with equal frequency [25].

As shown in the table 3 and 4 , the Karl Pearson correlation coefficient method turns to be positive relation between the post test scores with frontalis and mentalis respectively. This correlation coefficient method is mainly used to find whether any relationship exists between the amplitudes of the muscles and the SFGS scores. The $p$ value is insignificant $(p=0.3241)$ for Frontalis pretest score and relatively significant $(p=0.0375)$ for post-test score. The $p$ value is again insignificant $(p=0.9797)$ for Mentalis pretest score and relatively significant $(p=0.0268)$ for post-test score. In a similar study by Mats Engstrom, shows a weak correlation initially among the values of frontalis, nasalis and mentalis. These muscles also show a strong correlation at the time of follow-up examinations. This holds true with our results that at the pre test scores of frontalis and mentalis shows no correlation and post test scores are positively correlated and there exists a correlation between the amplitudes and the SFGS scores.

The third visit or the follow-up visit was not considered in the study as although after explaining the procedure to the subjects none of the subjects did come for the follow-up visit.

Recovery from the first degree of injury in Bell's palsy is always complete, provided the nerve is relieved of compression. In a study done by Lars Thomander and Erik Stalberg, their result shows the early start of the clinical and electrophysiological improvement may indicate that a temporary conduction block was present. Yeo and Lee in 2007 suggested that $96 \%$ of Bell's palsy recovered spontaneously. Therefore, in our study we correlated electrophysiological readings with the clinical outcome scale.

Furthermore, the multiple regression analysis was used as shown in table 5 and 6 , for the frontalis and the mentalis amplitudes comparing with the pretest and the post test, respectively. The pretest and the post test $p$-values were found to be insignificant for the frontalis and mentalis amplitudes. Therefore, we found that there is no significant relationship between the frontalis and the mentalis muscle amplitudes individually with each other in the pre and post test SFGS scores.

As shown in the table 7 , the logistic regression analysis was also used. Logistic regression analysis was performed of posttest SFGC scores (low and high) by frontalis amplitude (low and high) and mentalis amplitude (low and high) scores. When the relationship between muscle amplitude and the post test SFGS scores were analyzed through this regression method, no significant difference between the amplitude of muscle and SFGS scores were found. The low and high scores were decided by considering the mean value and the low and high scores of that value. In our study, we assessed the prognostic value of the facial nerve CMAP ratio with the SFGS scores. The recovery rate was directly proportional to the CMAP ratios above $10 \%$ were of significance.

The comparison of the pretest and the posttest of SFGS scores by paired t-test were also conducted as shown in the table 8 . The overall paired $t$ value was found to be -4.8318 and the $p$ value is 0.00001 , which is significant. Overall, our results posttest SFGS scores signify improvement is there from the initial readings.

We also found, in our study that the MDC value (minimal detectable change) at $95 \% \mathrm{Cl}$ was $3.786 \mathrm{H}^{\prime \prime} 4$. Therefore, any change of 4 in the score of SFGS can be taken as a minimal detectable change. Our results shows that the mean gain obtained is of 10 , which is much higher than 4 and suggests that there is a marked clinical difference present. This is in reference with the data of the cases that have followed up until the second NCV. This analysis is based on the 38 subjects out of 58 subjects.

Better prognosis is indicated if the CMAP of the affected side is more than $10 \%$ of the normal side amplitude. Poor prognosis is indicated if the amplitude of the affected side is less than $10 \%$ of that of normal side. Comparison of the $10 \%$ amplitude of the normal side (greater than or less than $10 \%$ ) with the muscle is shown in the table 9. Out of 58 patients, for the frontalis muscle 57 patients had chances Int J Physiother Res 2022;10(1):4102-10. I ISSN 2321-1822 
of better prognosis and 1 patient whose amplitude of the affected side less than $10 \%$ of the normal side showed poor prognosis. For the mentalis muscle, all 58 subjects had chances of better prognosis with the affected side amplitude greater than $10 \%$ of the normal side respectively.

As in Table 10, it depicts the correlation between frontalis and mentalis first reading with gain of pre and post test scores of SFGS. In this table, we took the 38 subjects who came on the $20^{\text {th }}$ day (second visit) and their first and the second readings were noted accordingly. The purpose of this analysis was predicting the prognosis with help of the NCV readings to that of SFGS scores (pre and post test). Accordingly the frontalis $p$ value was not significant and the mentalis $p$ value was a statistically significant result. Therefore, we found that mentalis is a good predictor of prognosis as compared to frontalis, in patients with Bell's palsy.

Overall, from our research by performing NCVs for two muscles i.e., frontalis and mentalis will focus on prognosis of each muscle individually, irrespective of the other. We believe it is because frontalis and mentalis belong to two different branches of facial nerve. Hence, if one branch of the facial nerve recovers, other may or may not recover.

NCVs have been proved to be an important test within the scope of the therapist. Nonetheless, the clinical training protocol should receive the same rigorous thought, study and practice as with the NCV readings on the routine basis.

\section{CONCLUSION}

This study was conducted to correlate, the SFGS scores which is a clinical outcome measure with that of NCV readings which is an investigative procedure, to predict the outcome of the patients with Bell's palsy. There were a total of 58 patients included in the study out of which the majority of patients did come for the follow-up second visit. SFGS score was noted initially and NCV was performed for the frontalis and mentalis muscle respectively. They were also undergoing a routine physiotherapy treatment in the course of the study. The patients were reviewed on the $20^{\text {th }}$ day, where NCV was performed and the SFGS score was noted again. The comparison was made between the initial scores and the change in the amplitude from the previous reading. Also, we were able to predict the prognosis of the patients with Bell's palsy who shall recover completely or partially. There was a positive correlation established between the NCV readings and the SFGS scores after the routine physiotherapy treatment.

Results showed a strong positive correlation of the CMAP amplitude ratio and the SFGS scores post treatment using Karl Pearson's correlation coefficient method. When multiple regression analysis was used we found that there is no relationship between the frontalis and mentalis amplitudes and both stands as independent variables. $99 \%$ of the patients had affected side amplitude greater than $10 \%$ indicating chances of better prognosis and only 1 patient had frontalis amplitude less than $10 \%$ indicating chances of poor prognosis.

To summarize, the NCV is a well-adapted for routine diagnostic and prognostic purposes in Bell's palsy. The results can easily be quantified and have sufficiently high accuracy for monitoring the nerve function. The findings can be used for early prognostication of recovery, thus permitting early detection of patients requiring therapy.

\section{ACKNOWLEDGEMENTS}

Above all, I would like to thank God for having blessed me and giving me all I need. My sincere thanks to Dr. Ravi Savadatti, Principal of S.D.M College of Physiotherapy for helping me in making this research a successful venture. I wish my deep sense of gratitude to Dr. C. G. Prashanth, Assistant Professor and PG Guide, S.D.M. College of Physiotherapy, for providing me with constant guidance for his most valuable suggestions. I would also like to thank the Neurophysicians and ENT department of SDMCMSH without whom, I would not have been able to complete the task. Very special thanks to my Parents and Family, for trusting in my abilities. Finally, I would like to thank all the subjects who participated in this research, without whom 
this study would have been impossible.

\section{Conflicts of interest: None}

\section{REFERENCES}

[1]. Tiemstra J., and Khatkhate N., Bell's Palsy: Diagnosis and Management. Oct.2007; 76(7): 997-1002.

[2]. Beck D. and Hall J.,Evaluation of the facial nerve via electroneuronography (ENoG). March 2001; 54(3).

[3]. Finsterer J., Management of peripheral facial nerve palsy. Mar.2008; 265:743-752.

[4]. Neely J., Cherian N., Dickerson C., Nedzelski J., Sunnybrook facial grading system: Reliability and criteria for grading. May 2010; 120(50):1038-1045.

[5]. Ross B., Fradet G., Nedzelski J., Development of a Sensitive Clinical Facial Grading System. March 1995.

[6]. Brenner M. and Neely J., Approaches to Grading Facial Nerve Function. 2004; 18(1):1535-2188.

[7]. Kimura J., Electrodiagnosis of the Cranial Nerves. Mar 2006; 15: 2-12

[8]. Kimura J., Principles and Pitfalls of Nerve Conduction Studies. Mar 1984; 16: 415-429.

[9]. Tojima H., Measurement of Facial Nerve Conduction Velocity and Its Application to Patients with Bell's Palsy. 1987;104(446):36-41.

[10].Schularick N., Mowry S., Soken H., Is Electroneurography Beneficial in the Management of Bell's Palsy? May 2013;1066-1067.

[11]. Venogopal M. ,Rajan S., Suma R., Thomas S., Etiopathogenesis of lower motor neuron facial palsy. Our experience, April 2011; 17(2).

[12]. Kumar P., Raymond A., The use of nerve conduction studies in determining the short-term outcome of Bell's palsy. Mar.2003; 58(1):69-78.

[13].Kitisomprayoonkul W, Sirileartthananon P, Chaiwanichsiri D., Electrodiagnosis and recovery of facial paralysis at King Chulalongkorn Memorial Hospital. Oct.2007; 90(10):2198-2203.

[14]. Yamout B., Zaytoun G., Nuweihud I., The role of facial nerve conduction studies and electromyography in predicting the outcome of Bell's palsy. July 1997; 4(4):348-351.
[15].Dumitru D, Walsh N E, Porter LD. in Electrophysiologic evaluation of the facial nerve in Bell's palsy. A review. August 1988; 4:137-144.

[16]. Ozgur A., Semai B., Hidir U., Mehmat Fatih O., Tayfun K., Zeki O., Which electrophysiological measure is appropriate in predicting prognosis of facial paralysis? Dec.2010; 112(10):844-848.

[17]. H. Z. Joachims H., Bialik V., Eliachar I., Early diagnosis in Bell's palsy. A nerve conduction study. Oct.1980; 90(10):1705-1708.

[18]. Jackson G., Doersten P., The Facial Nerve, 83(1): 179-195.

[19]. Gantz B., Rubinstein J., Gidley P., Woodworth G., Surgical Management of Bell's Palsy. Aug 1999; 109:1177-1188.

[20].Thomas B., Elin M., Mats E., Malou H., Hadziosmanovic N., Lars J., The Effect of Study Design and Analysis Methods on Recovery Rates in Bell's Palsy. Oct.2009; 119:2046-2050.

[21]. Danielidis V., Skevas A., Cauwenberge P., Vinck B., A comparative study of age and degree of facial nerve recovery in patients with Bell's palsy. November 1999; 256:520-522.

[22]. Kar N, Banerjee S. K, Prediction of recovery of Bell's palsy from clinical manifestations. October 1992; 10:267-269.

[23]. Smith I., Heath J., Murray J., Cull R., Idiopathic facial (Bell's) palsy: a clinical survey of prognostic factors. February 1988; 13:17-23.

[24]. Wenkebach P, Fikentscher R, Skurczynski W., Agedependent prognosis of ischemic facial paralysis. 1983; 64:168-169.

[25]. Gilden D., Clinical Practice: Bell's palsy. Sep.2004; 351(13):1323-1331

How to cite this article: Neha Patel, Pragna Landge. To Study the Correlation of Nerve Conduction Velocity Studies and The Outcome of Subjects with Bell's Palsy on Sunnybrook Facial Grading Scale. Int J Physiother Res 2022;10(1):4102-4110. DOI: 10.16965/ijpr.2021.211 